\title{
Development of a portable SiPM scintillator tracker for
} cosmic rays

\author{
R. Pillera, ${ }^{a, b, *}$ C. Altomare,${ }^{b}$ E. Bissaldi, ${ }^{b, c}$ S. De Gaetano, ${ }^{b, c}$ G. De Robertis, ${ }^{b}$ \\ P. Dipinto, ${ }^{b}$ L. Di Venere,${ }^{b, c}$ M. Franco, ${ }^{b}$ F. Gargano, ${ }^{b}$ F. Giordano,,${ }^{b, c}$ N. Lacalamita, ${ }^{b}$ \\ F. Licciulli, ${ }^{b}$ F. Loparco, ${ }^{b, c}$ S. Loporchio, ${ }^{b}$ F. Maiorano, ${ }^{b}$ S. Martiradonna, ${ }^{b}$ \\ M. N. Mazziotta, ${ }^{b}$ M. Mongelli, ${ }^{b}$ M. G. Papagni, ${ }^{b}$ C. Pastore, ${ }^{b}$ M. Rizzi, ${ }^{b}$ D. Serini ${ }^{b}$ and \\ R. Triggiani ${ }^{b}$ \\ a Politecnico di Bari, Department of Mechanics, Mathematics and Management, \\ via Orabona, 4, I-70125 Bari, Italy \\ ${ }^{b}$ Istituto Nazionale di Fisica Nucleare, Sezione di Bari, \\ via Orabona 4, I-70126 Bari, Italy \\ ${ }^{c}$ Dipartimento di Fisica “M. Merlin”, dell'Università e del Politecnico di Bari \\ via Amendola 173, I-70126 Bari, Italy \\ E-mail: roberta.pillera@ba.infn.it
}

A crucial aspect for outreach activities in cosmic-ray physics is the ability to bring the audience in contact with the experiments and let them visualize the underlying physics. A possible option is the use of portable detectors, which can be easily transported and operated in the various places where outreach activities take place (schools, theaters, etc.), and are equipped with a fast data acquisition system for real-time event visualization. We have developed a tracker for cosmic rays composed of scintillator bars with embedded wavelength shifting (WLS) fibers coupled to Silicon Photomultipliers (SiPMs). The SiPMs are read out with Caen DT5702 Front-End boards and the data acquisition is performed with custom $\mathrm{C}++$ /python based software. The DAQ is controlled by a Raspberry Pi 4 single computer board, equipped with a GPS for precise timing and position information and a Sense HAT board for environmental monitoring. This results in a simple, portable system allowing online track visualisation and cosmic ray rate measurements.

$37^{\text {th }}$ International Cosmic Ray Conference (ICRC 2021)

July 12 th - 23rd, 2021

Online - Berlin, Germany

\footnotetext{
${ }^{*}$ Presenter
} 


\section{Introduction}

The direct contact of the audience with experiments is an important aspect for outreach activities in cosmic-ray physics, in order to allow them to visualize the underlying physics. The use of portable detectors, equipped with a fast data acquisition system for real-time event visualization is a possible option. We are developing a tracker for cosmic rays composed of scintillator bars with embedded wavelength shifting (WLS) fibers coupled to Silicon Photomultipliers (SiPMs).

Fig. 1 shows a CAD scheme of the detector. The detector is composed by $4 \mathrm{x}-\mathrm{y}$ planes, each consisting of 2 layers of 8 scintillator bars, disposed along one of the two axes. Each bar has an embedded WLS fiber, with 2 SiPMs connected at both ends. The readout is performed using the Caen DT5702 32 channel frond end board (FEB) [1], and each FEB can read 2 layers. The implementation of a new custom 128 channel FEB, which can read up to 8 layers, is in progress. The data acquisition (DAQ) is controlled with a Raspberry Pi 4 B+ card [2] using the ethernet protocol. The telescope has been built using materials already available in our laboratory. This detector is planned to be used for several purposes:

- Outreach activities;

- Educational particle physics laboratories;

- Test bench for custom designed FEB;

- General purpose tracker.

\section{Detector hardware}

Fig. 2 shows the scintillator bars and WLS fibers. The bars are made of extruded plastic scintillator. Their dimensions are $20 \times 1.9 \times 1.5 \mathrm{~cm}^{3}$ and they have a $4 \mathrm{~mm}$ diameter hole to accommodate the WLS fiber. The peak emission of the scintillator material is in the blue region. We use the BCF-92 fibers produced by Saint-Gobain, which shift blue to green light. In Fig. 3 a) we

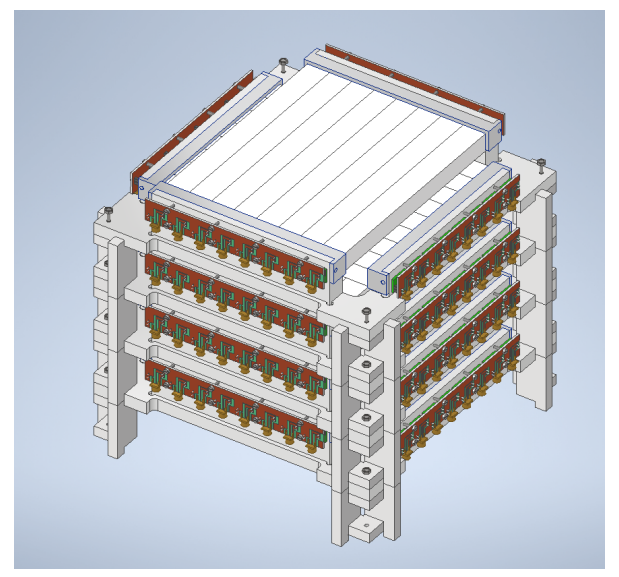

Figure 1: CAD scheme of the detector. The detector is composed by $4 \mathrm{x}-\mathrm{y}$ planes, each consisting of 2 layers of 8 scintillator bars, disposed along one of the two axes. 


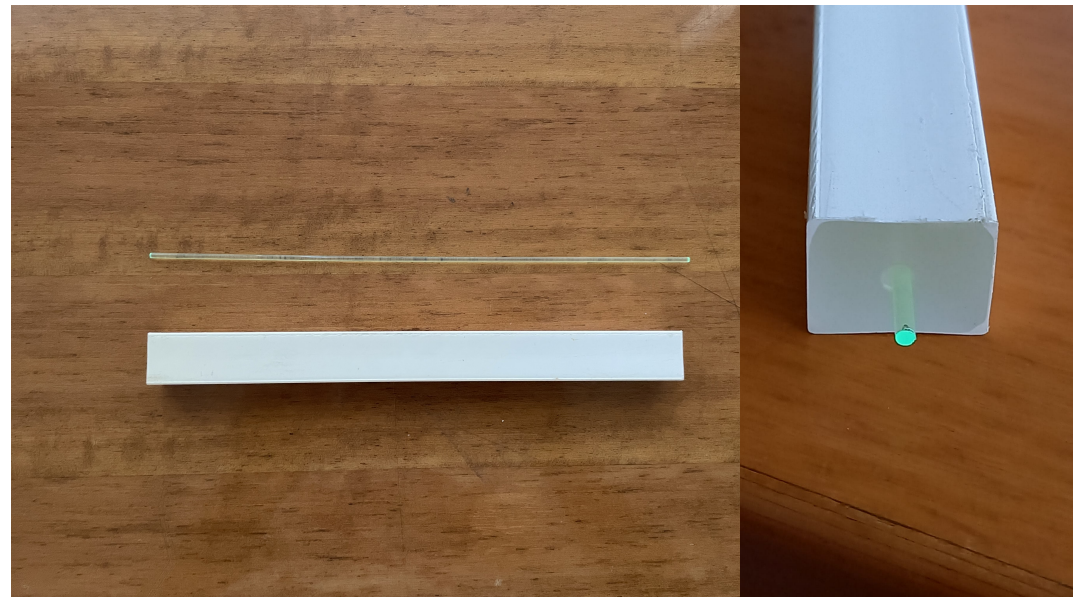

Figure 2: Scintillator bar and WLS fiber.

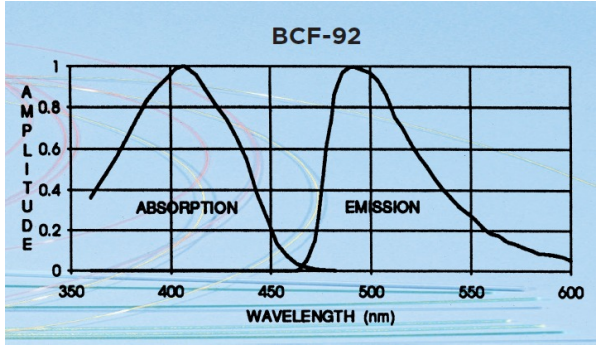

a)

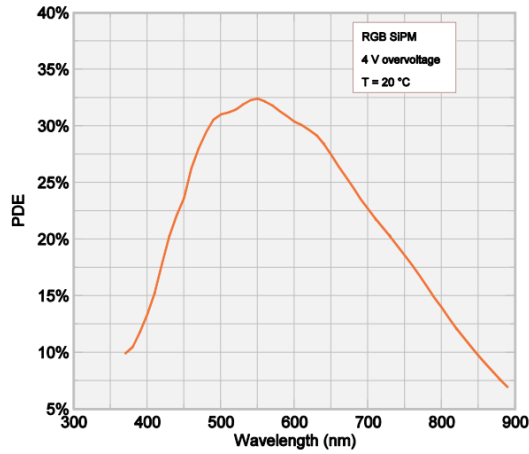

b)

Figure 3: a) Emission and absorption spectra of the Saint-Gobain BCF-92 WLS fibers [3]. Absorption and emission are peaked in the blue and green wavelength regions respectively. b) Photon detection efficiency (PDE) in RGB-SiPMs as a function of wavelength [4].

show their emission and absorption spectrum. The fibers have a diameter of $2 \mathrm{~mm}$ and a length of $21.5 \mathrm{~cm}$. They are slightly longer then the bars, in order to fit inside the mechanical support frame.

The photons exiting from the fiber are collected by SiPMs at their ends. We have used devices produced by AdvanSiD, sensitive to RGB light [4]. The main advantages of using SiPMs are their compactness, the low power consumption and the cost. They are available in two sizes, with $1 \times 1 \mathrm{~mm}^{2}$ and $3 \times 3 \mathrm{~mm}^{2}$ active area. They have $40 \mu \mathrm{m}$ side micro-cells, and a breakdown voltage $V_{b d}=28 \mathrm{~V}$. The plot in Fig. $3 \mathrm{~b}$ ) shows the SiPM photon detection efficiency (PDE). The WLS emission peak at $492 \mathrm{~nm}$ matches the SiPM maximum PDE of about $30 \%$ at $4 \mathrm{~V}$ overvoltage.

\section{DAQ system}

Having a portable detector as a goal, we chose for DAQ control a Raspberry Pi 4 B+ card [2], with 8GB RAM. We provided a large SD memory card for data storage. The communication with the FEB is done with an ethernet connection. Fig. 4 shows a block diagram of the system. It has 




Figure 4: Block diagram of the system.

been equipped with a GPS (L80-M39), in order to provide a pulse-per-second (PPS) signal to the board. This allows the generation of a relative timestamp per event, that is required to cross match data in multiple board DAQ. The GPS is controlled by the Raspberry Pi via a USB connection and the PPS is given to the board in TTL standard through a coaxial cable with a LEMO connector. Besides GPS information, that is available in the DAQ, a SENSE HAT for slow monitoring of temperature and asset connected through the GPIO pins is foreseen [5], in order to allow online reconstruction of the directions of cosmic rays in an absolute reference frame.

For SiPM powering and readout we use the commercial Caen DT5702 32 channel FEB [1]. The board is based on the CITIROC front-end ASIC by Weeroc [6]. It is possible to implement two trigger logics. The first one requires the coincidence between the signals from the SiPMs at the two ends of any individual bar. This trigger logic is used to select cosmic-ray muon events. If a muon crosses a scintillator bar, a signal above the threshold from both the SiPMs connected at its ends is expected. The second trigger logic requires a signal from any channel, and this mode of operation is used for pedestal calibration runs.

\section{Prototype}

We have assembled the first of the $4 \mathrm{X}-\mathrm{Y}$ planes foreseen in the final configuration. Fig. 5 shows some pictures of the prototype. Fig. 5a) shows the scintillator bars placed inside the mechanical frame. In Fig. 5b) we can see the fibers emerging from the holes in the scintillator bars. The SiPMs are placed in front of the fibers on custom designed boards (the back and front view of these boards are shwn in Fig. 5c) and Fig. 5d) respectively). The SiPMs are connected to the DAQ board with MCX cables and a custom 32 channel connector. The frame and the boards were designed and manufactured by the technical staff of the mechanics and electronics workshops of our department. In our prototype the fibers coupled to the bars of plane 0 (X direction) are readout with $3 \times 3 \mathrm{~mm}^{2}$ and $1 \times 1 \mathrm{~mm}^{2}$ SiPMs on the two sides, while those of plane 1 (Y direction) are readout with $3 \times 3 \mathrm{~mm}^{2}$ SiPMs on both sides. The detector is placed inside a dark box, while the DAQ board and the Raspberry Pi are placed outside for cooling purposes. The final portable device will be equipped with a 7" touch display by Raspberry Pi. 


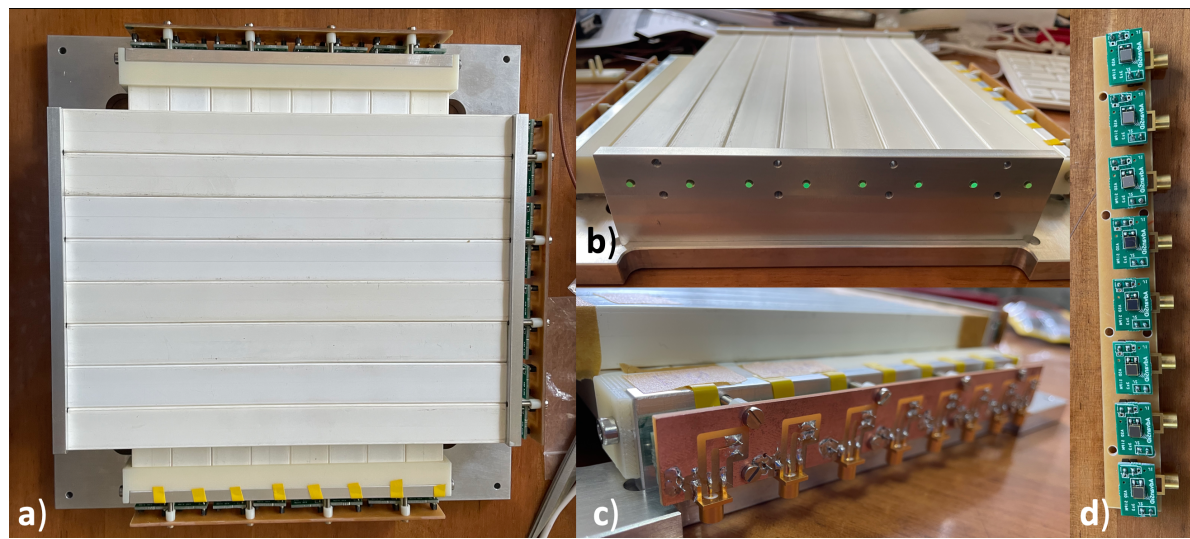

Figure 5: Prototype of a single X-Y plane.

\section{Software}

The data acquisition software is based on the $\mathrm{C}++$ library provided by Caen, implementing the communication with the DT5702 board, called FEBDTP [7].

We developed a python-based toolkit to easily customize the interface with the user. This toolkit is based on a python wrapper for the FEBDTP class, which was created using the Simplified Wrapper and Interface Generator (SWIG [8]). The main class of the toolkit helps the user to handle the features of the FEB, such as configuring the CITIROC parameters, enable/disable the channels in the trigger, change the trigger logic among those available in the DT5702 firmware, turn the HV on and off and perform the DAQ. A simple configuration file based on python dictionaries allows the full configuration of the board. This feature allows the user to manage the DAQ with simple python scripts. In addition, the toolkit is able to handle the external sensors connected to the Raspberry Pi, such as the GPS and the SENSE HAT.

We have also developed a Graphical User Interface (GUI) to operate the detector with the 7" touch display, without using mouse and keyboard. With this interface it is possible to:

- change configurations;

- check board connection and GPS status;

- execute calibration runs;

- execute cosmic-ray runs;

- activate the online event display visualization.

One of the goals of this tool is to perform a full online track reconstruction, and this feature will be implemented when more planes will be added to the prototype. When a cosmic-ray run is started from the GUI, a separate process is started using the python multiprocessing packet. This will call the DAQ function of the DAQ handling class, that is sharing data with the main process through a common queue. At the same time, the main application will update the event display. Due to the event processing time and graphics, approximately one every ten events are displayed. The DAQ 




Figure 6: Frame of DAQ GUI application.

can be stopped manually by the user pushing a "STOP" button. A screenshot of the application is shown in Fig. 6.

\section{Cosmic-ray runs}

In Fig. 7 we show the ADC count distribution from an individual channel. The distribution exhibits a peak at low counts, corresponding to the pedestal, while the bulk of cosmic-ray events are in the region around the second peak. As mentioned in Sec. 3, a bar is considered to be hit if the signals from both channels at each end of the fiber exceed a threshold, which has been set at $5 \sigma$ above the pedestal. For each channel the value of $\sigma$ is obtained from a Gaussian fit of the pedestal performed in dedicated runs.

The plot in Fig. 8 a) shows the distribution of the number of active bars per event. The blue distribution is obtained considering all planes, and it is peaked at two bars, meaning that most selected events correspond to cosmic rays, which cross both the $\mathrm{x}$ and y layers, hitting one bar per layer. Most of the events with higher bar multiplicities correspond to muons, whose incoming

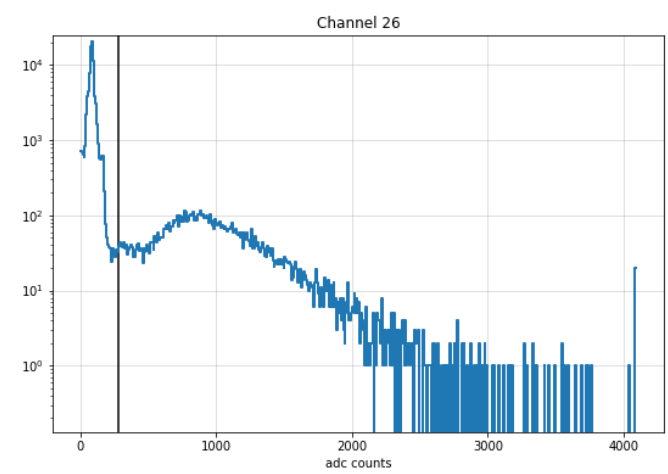

Figure 7: ADC count distribution of an individual channel. The vertical black line indicates the event selection threshold at $5 \sigma$ above the pedestal. 


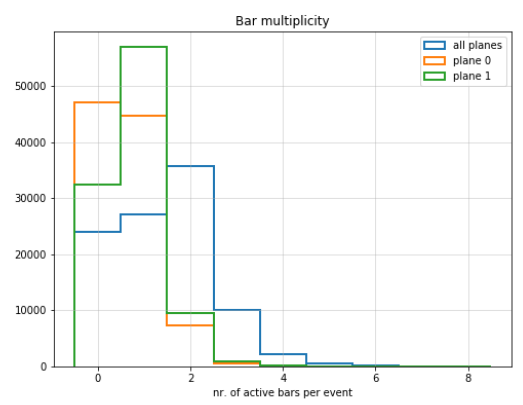

a)



b)

Figure 8: a) Bar multiplicity distribution. The blue distribution is obtained considering all planes, the orange and green ones taking only plane 0 and plane 1 respectively. b) Hit map. The bar nr. 7 has no hits due to a dead channel.


Figure 9: Examples of event displays. Top: multiple muon candidate; middle: muon with very high inclination angle candidate; bottom: muons with shower candidate.

direction is tilted with respect to the z-axis, which typically cross two adjacent bars in a plane (see the middle panel of Fig. 9). However, we also find some events with multiple muons. In this case, the hit bars in a plane are not adjacent (see the top panel of Fig. 9). There is also a small fraction of muons accompanied by showers initiated in the concrete layers above our laboratory (see the bottom panel of Fig. 9). In these events, clusters of two or more adjacent bars in a plane are fired. The green curve in Fig. 8 a) shows the multiplicity distribution for plane 1, that is peaked at one bar as expected, whereas the orange curve corresponds to plane 0 . We can see that the efficiency of 




Figure 10: Total ADC charge distribution for a cosmic-ray run. It follows a Landau distribution as expected.

plane 0 is lower, mostly due to a dead channel and the use of the smaller SiPMs on one side. Fig. 8 b) shows the map of the hit bars. We see that there are no hits in bar nr. 7 due to a dead channel.

In Fig. 10 we can see the total event charge distribution. It is obtained by summing the charges of all ADC channels corresponding to the active bars. As expected it follows the Landau distribution, even though the gains of individual channels have not been equalized.

\section{Conclusions}

We have started to build a muon cosmic-ray tracker prototype for educational purposes. The next step is to complete the setup with more planes and to release the software and a full documentation. An upgrade with a custom DAQ board using the PETIROC front-end ASIC [9] is also in progress. With this new board it will be possible to read all planes with a single board, use the gps for single event timestamp generation and a more flexible trigger logic.

\section{References}

[1] https://www.caen.it/products/dt5702/

[2] https://wWw.raspberrypi.org/products/raspberry-pi-4-model-b/?variant= raspberry-pi-4-model-b-8gb

[3] https://www.crystals.saint-gobain.com/sites/imdf.crystals.com/files/ documents/sgc-scintillation-fiber_0.pdf

[4] https://advansid.com/attachment/get/up_54_1432741139.pdf

[5] https://wWw.raspberrypi.org/products/sense-hat/

[6] https://www.weeroc.com/products/sipm-read-out/citiroc-1a

[7] https://wWw.caen.it/download/?filter=DT5702

[8] http://www.swig.org/index.php

[9] https://www . weeroc.com/products/sipm-read-out/petiroc-2a 